“ C 2019 IEEE. Personal use of this material is permitted. Permission from IEEE must be obtained for all other uses, in any current or future media, including

reprinting/republishing this material for advertising or promotional purposes, creating new collective works, for resale or redistribution to servers or lists, or reuse of any copyrighted component of this work in other works." 


\section{Enabling Design of Middleware for Massive Scale IOT-based Systems}

\author{
Zenon Chaczko \\ Faculty of Engineering and IT, \\ University of Technology, Sydney, \\ NSW, Australia \\ zenon.chaczko@uts.edu.au \\ Christopher Chiu \\ Faculty of Engineering and IT, \\ University of Technology, Sydney, \\ NSW, Australia \\ christopher.chiu@uts.edu.au
}

\author{
Ryszard Klempous \\ Faculty of Electronics and Computing \\ Wroclaw University of Technology, \\ Wroclaw, Poland \\ ryszard.klempous@pwr.wroc.pl \\ Konrad Kluwak \\ Faculty of Electronics and Computing \\ Wroclaw University of Technology, \\ Wroclaw, Poland \\ konrad.kluwak@pwr.edu.pl
}

\author{
Jerzy Rozenblit \\ Dept. of Elec. \& Comp. Engineering, \\ The University of Arizona \\ Tucson, Arizona 85721, USA \\ ir@ece.arizona.edu
}

Ito Atsushi

Utsunomiya University,

7-1-2 Yoyo, Utsunomiya,

Tochigi 321-8505, Japan

at.ito@is.utsunomiya-u.ac.jp

\begin{abstract}
Recently, the Internet of Things (IoT) technology has rapidly advanced to the stage where it is feasible to discover, locate and identify various smart sensors and devices based on the context, situation, their characteristics and relevancy to query for their data or control actions. Taking things $\overline{\bar{y}}$ a step further when developing Large Scale Applications requires that to-overeome-two serious issues be overcome. The first issue is to find a solution for data sensing and collection from a massive number of various ubiquitous devices when converging these into the next generation networks. The second important issue is to deal with the "Big Data" that arrive from a very large number of sources. This research emphasizes the need for finding a solution for a large scale data aggregation and delivery. The paper introduces biomimetic design methods for data aggregation in the context of large scale IoT-based systems.
\end{abstract}

Keywords- Middleware, Ubiquitous Systems, Massive-scale systems, Internet of Things, $5 G$ Communications

\section{AN OVERVIEW OF MIDDLEWARE DESIGN FOR IOT CONCEPTS}

The IoT domain represents some of the greatest research challenges and opportunities that have appeared in the modern era. New emergent technological advances related to the wireless smart sensor and actuators, autonomic computing [1], Artificial Intelligence (AI), middleware [2], communication systems and protocols, engineering standards and the modern fifth generation mobile networks (5G) are driving a new paradigm whereby sensors and actuators are handled as a common infrastructure with the functionality to provide concurrent and multiple services, and smart data aggregation to variety of end users [3-4].

New The challenges of new-communication technologies in the realm of IoT will pose numerous challenges in the multimedia sphere. The roll out of $5 \mathrm{G}$ along with IoT will allow for immediate connections between machine to machine (M2M) systems, as well as near real-time interactivity for users. Practical considerations of $5 \mathrm{G}$ are factored into the needs of multimedia connectivity and interactivity.

Also just as importantly are,-the effects and significance that IoT with $5 \mathrm{G}$ places on future demand, based on the previous experiences of mobile cellular connectivity. New services such as Virtual Reality (AR) and Augmented Reality (AR), as well as multi-person video conferencing need to be considered in the scope of IoT middleware, along with the technical network architecture.
This approach differs with the concept of dedicating devices being logically and physically fixed within the dedicated computer and telecommunication infrastructures. Significant research work still needs to be done to adapt modern middleware models, protocols and standards, data aggregation and sensor fusion methods as well as the data delivery mechanisms to modify the way IoT devices could become an integral part of a common public infrastructure. This is because the rapid rise of new communication solutions will demand suitable software infrastructures to support adequate M2M connectivity with minimal interruptions or disruptions to existing operations.

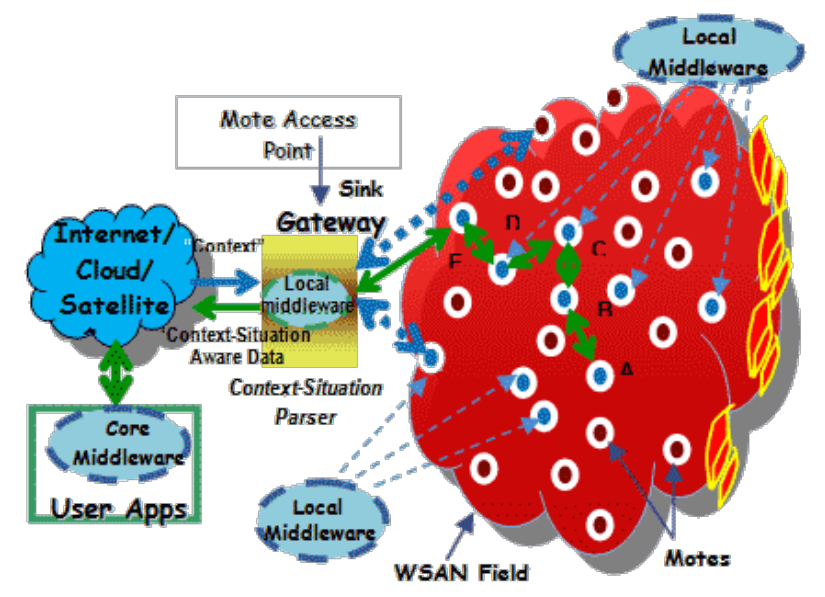

Fig. 1. Middleware for the IoT-based System

One of the key issues to be solved in the presented work is providing a feasible model of architecture for implementation and design of a new construct of software-driven infrastructure that is referred to as a biomimetic model of middleware (BMM) [5]. A typical topology and localisation of middleware components in IoT-based software application is depicted in Figure 1, consisting of the IoT motes, core middleware and local middleware, along with the Internet.

In IoTs, the Mote Access Point (MAP) devices can play dual roles: these devices are data sinks for sensors and a control sinks for actuators that form segments of the IoT, as these segments partition the separate responsibilities that each component has to operate effectively. The MAP devices represent intelligent gateways, or essentially access points with routing functions, that host the contextual-situation data processors/parsers at the boundary of sensor/control of IoT motes. 
In this research, a new paradigm of biomimetic middleware for IoT-based systems is introduced, together with the supporting adaptive data aggregation models. The biomimetic middleware allows a new generation of multipurpose sensor-actuator networks and services to become a viable solution [5]. This research work discusses various design challenges of IoT middleware. Innovations involve the vision for the advanced of large scale, software intensive IoTbased systems for modern computing and the connections establishing machine-to-machine communication.

This work is organized into the following sections. After the introduction, the paper proposes a new biomimetic model of middleware (BMM) and reviews adaptive data aggregation techniques. Afterwards, design aspects of biomimetic middleware solution that actively provide deployment and development of context aware, secure, heterogeneous, largescale and flexible system applications [6]. Once an illustration establishes the feasibility, as well as viability of implementing the biomimetic middleware for a large-scale IoT-based system, it is then possible to conceive the deployment of such infrastructures from a broader perspective, especially that of Software Defined Networking (SDN) solutions that will drive $5 \mathrm{G}$ mobile connectivity.

\section{BIOMIMETIC MODELS OF MidDLEWARE}

The consideration of advancing research in middleway modern fields of middleware includes the following biomimetic concepts, along with their analogous system solutions [7] in Table I:

TABLE I. MODERN BIOMIMETIC SOLUTIONS

\begin{tabular}{|l|ll|}
\hline \multicolumn{3}{|c|}{ Modern Biomimetic Solutions } \\
\hline \multicolumn{1}{|c|}{ Technical Advancement } & \multicolumn{1}{c|}{ Analogous Solution } \\
\hline $\begin{array}{l}\text { Manufacturing } \\
\text { Advancements }\end{array}$ & $\bullet$ & Kirigami \\
& & 5D Printing/Additive \\
& Manufacturing Infrastructures \\
\hline & $\bullet$ & Containers/Funtainers FaaS \\
Cloud-based & $\bullet$ & Artificial Intelligence-based \\
Infrastructures & & MLaaS/AIaaS platforms in the \\
& & cloud \\
\hline & $\bullet$ & Intel AI \\
& $\bullet$ & H2O \\
Open-Learning & $\bullet$ & RStudio \\
Frameworks & $\bullet$ & Python Frameworks: Pytorch \\
& & and Torch \\
& $\bullet$ & TensorFlow \\
& $\bullet$ & Caffe, Caffe2 \\
& $\bullet$ & Chainer, CNTK \\
&
\end{tabular}

As shownean be observed in Table I, modern technological advancements are driving biomimetic design in various forms. This is especially evident in the following arenas:

\section{A. Manufacturing Advancements}

\section{1) Kirigami}

The Japanese art of unfolding structures into elaborate constructs. Traditionally, the folding points are placed strategically to ensure a highly compressed structure in its folded form, but when unfolded reveals its full form that serves its true intention or function.

The uniqueness of this approach is beneficial where confined or restricted IoT environments place specific demands where the solution can be compressed into its stored form when not in use, or when a different structure is required to serve its purpose.

\section{2) $5 D$ Printing/Additive Manufacturing:}

The rise of additive manufacturing has led to the construction of complex systems that could not be built using traditional assembly manufacturing systems, leading to sophisticated, unique designs that drive modern rapid, instantaneous manufacturing advancements.

Rapid prototyping is a major benefit of such solutions, meaning that new devices can be launched into the IoT field with minimal delay and outlay costs, facilitating a seamless infrastructure rollout that is highly efficient and applicable in its construction.

\section{B. Cloud-based Services and Infrastructures}

\section{1) Containers/Funtainers as a Service (FaaS)}

These container-based structures are gaining rapid interest in the education industry, as it allows users to experiment and try out new ideas in a safe, encapsulated environment, free from distractions and constraints.

FaaS platforms are prominent in their ability for rapid experimentation, which serves well in IoT structures where rapid testing and results can be quickly gathered and analyzed, with new algorithms developed to test and deploy in the IoT field.

2) Machine Learning/Artificial Intelligence as a Service (MLaaS, AIaaS)

These AI-driven platforms in the cloud have been widely adopted in industry and research, because the low cost of entry has been a significant driver. Technology giants such as Amazon Web Services, Google Cloud and Microsoft Azure are commonplace offerings with AI capability.

MLaaS and AIaaS has rapidly shifted from general computing services, towards specific dedicated Application Specific Integrated Chipset (ASIC) and Systems on a Chip (SOC) solutions that seek to optimize computing efficiency, and hence lower the total cost of operations on the end user.

IoT middleware solutions are beneficiaries from MLaaS and AIaaS, as dedicated AI solutions can be deployed in a remote location away from the IoT cloud, where dedicated computing resources are specifically tailored for operational and functional efficiency.

\section{Open-Source Learning Frameworks:}

The main advantage of open source learning frameworks is the collaborative nature of these platforms. Institutions can contribute as required to uniquely customize $\mathrm{AI}$ algorithms to suit a specific domain or research interest, or in our research interest, IoT middleware solutions.

Examples of Open Source Learning Frameworks include vendor driven initiatives such as Intel AI, along with various community solutions including $\mathrm{H} 2 \mathrm{O}$, RStudio, Python Frameworks, Pytorch and Torch, TensorFlow, Caffe, Caffe2, Chainer, CNTK, DSSTNE, DyNet, Gensim, Gluon, Keras, Mxnet, Paddle and BigDL [9].

The use of general Machine Learning (ML) methodologies has been typically driven by agriculture, medicine, sport, entertainment, social media and other similar industries. The emphasis on working assets with AI becomes uniquely placed when IoT infrastructures are involved, as the management, 
configuration and routing of IoT motes must be efficiently performed to conserve the limited energy resources inherent in IoT networks.

Furthermore, IoT middleware can be tasked to perform more specific operations such as pattern recognition enhancement and technical advancement in commercial and industry applications. This is especially beneficial in industry sectors such as security surveillance where humans can be augmented with AI-driven recognizers to assist in the daily operations of workers where repetitive or laborious tasks can lead to fatigue.

Pattern recognition requires Computer Vision (CV) enhancement with best practice, with a focus on the advancing pace of technical advancements, particularly where the computing capability of mobile, power constrained devices is reaching a point where basic AI operations can take place on the field. This means a hierarchical middleware infrastructure must factor the different AI demands placed upon it, where smaller operational tasks are performed on-field, and more complex big data operations take place off-site at the MLaaS or AIaaS - as a case in point [7-8]:

\section{1) On-site/field: Local AI Operations in IoT}

Local AI operations in IoT are most suitable for unsupervised machine learning operations, such as neural networks or Self-Organized Maps (SOM). This is because the computational and energy resources in the IOT gateway are constrained, as well as the data repository resources, mainly being the backend database.

The middleware will need to prioritize unsupervised learning tasks on the local IoT operation, because remote AI operational needs should focus on more heavy computational requirements [10]

\section{2) Off-site/field: Remote AI Operations in IoT}

Remote AI operations in IoT are most suitable for supervised machine learning operations, such as genetic algorithm (GA) programming tasks. MLaaS or AIaaS systems are most suitable for such concerns, because the computational and energy resources are far more generous for such tasks [11-12].

Tailored MLaaS or AIaaS solutions will typically contain denormalized database structures suitable for the long-term monitoring and archiving of IOT data, that is most essential in the long-term trend analysis tasks that are performed in such scenarios

\section{APPLICABILITY OF BiOMIMETIC MidDLEWARE SOlutions IN THE FIELD}

\section{A. Role of IoT and Motion Capture Technology}

The applicability of biomimetic middleware is evident in IoT applications where tracking of human motion is important. As an evolving commercial field, many modern consumer technologies now adopt motion capture technologies, embedded directly in the hardware such as Microsoft Kinect, Sony SoftKinetic and Apple FaceID - all driving the future needs of VR and AR solutions in the consumer space.

The consumer space is an important driver, because this means that wide adoption of these technologies lead to lower build costs, and thus making a mainstream solution. Since IoT can be rapidly deployed with common consumer-based components, this means that total cost of ownership is dramatically reduced, and leading to the rapid momentum required to adopt such technologies in the IoT middleware infrastructure.
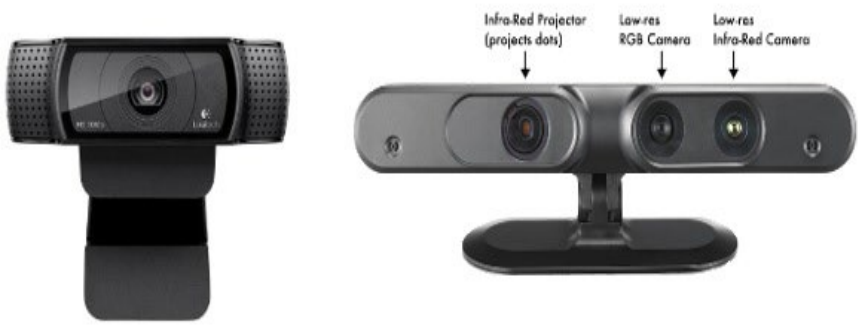

Fig. 2. Consumer Motion Capture Technologies (Left) RGB Web Camera, and (Right) Infrared ToF Depth Camera

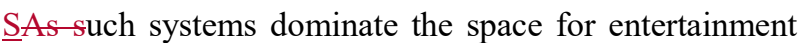
and gaming purposes as shown in Figure 2, including set-top units, arcading environments, multimedia and interactive advertising domains; therefore it becomes a natural fit for IoT solutions to also adopt motion capture technologies in the field as well for environmental monitoring applications.

Meanwhile, the professional workspace is-alse_gaining momentum as well. Sophisticated technologies with a high level of precision include vendor specific solutions such as GaitTrack, TekScan, Qualisys and Contemplas - to name notable vendors from North America and European Union. As these tools are prohibitive (cost-wise) in their initial outlay, such technologies also have recurrent maintenance costs, also limiting wider adoption.

Therefore, consumer motion capture technologies are most applicable for IoT middleware solutions, as broader adoption of M2M and IoT infrastructures are to take place with the eventual launch of 5G networking. Cost of replacement due to regular wear and tear must be minimized for adoption of such technologies to reach the mainstream, and thus overall accessibility to the public end user.

\section{B. Facilitating the end user in IoT Middleware}

The middleware infrastructure must be conceived to consider the design quality control when developing a economical and flexible platform for motion capture and annotation analysis. The bridging of the consumer space, mainly the multitude of motion tracking solutions for end users; along that of the closed, proprietary space of advanced professional motion capture solutions, is essential to provide practical, complementary applications that serve ubiquity as well as professional results into mainstream society.

Thus, any IoT solution incorporating biomimetic middleware along with motion capture must emphasize being agnostic to any technology vendor. An effective middleware solution must implement open source technologies and tools whenever possible, to ensure broad mainstream adoption, while also being able to incorporate new hardware solutions when they are launched.

Therefore, the middleware IoT solution must not be designed as a pure glue to bind logic components in one piece, but as a ubiquitous-driven platform that actively is facilitating in the integration of heterogeneous IoT components. This means that the reachability and wider adoption of the 
biomimetic middleware solution is accomplished - and thus provide an effective M2M layer for motion capture tool data sources, both consumer and professional, to be integrated in a seamless system.

\section{IoT Middleware Research Innovation}

The IoT middleware technology platform must be conceived to drive development in the key studies for applications such as motion capture, when possible in cooperation with academic institutions and commercial enterprises to achieve further innovation and research potential.

This is ultimately achieved by ensuring the IoT middleware platform is built with customizability in mind, irrespective of any industry sector or commercial imperative. Specific analysis where customization must take place should be at the MLaaS or AIaaS backend, to ensure that a logical separation takes place between IoT cloud and computational processing space.

The diversity in applications for IoT-driven motion capture solutions is vast and diverse, especially in the realm of the motion picture industry, television broadcasting, sports physiotherapy, healthcare and medical domains, aged/senior care management and biometric analysis of gait for a variety of applications.

In this paper, a focus application is tasked on applying a biomimetic approach to middleware for healthcare worker analysis, as a feasible approach must be undertaken to ensure work tasks in the middleware infrastructure is effectively segmented into practical, piecemeal operations. Thus, the case study will consider the needs of medical healthcare workers, and how IoT biomimetic middleware design can practically accommodate for such needs in conjunction with allied research professionals.

\section{Case Study: Healthcare Worker Analysis}

1) Introduction to Problem Space of Lumbar Lifting Analysis of Heavy Loads
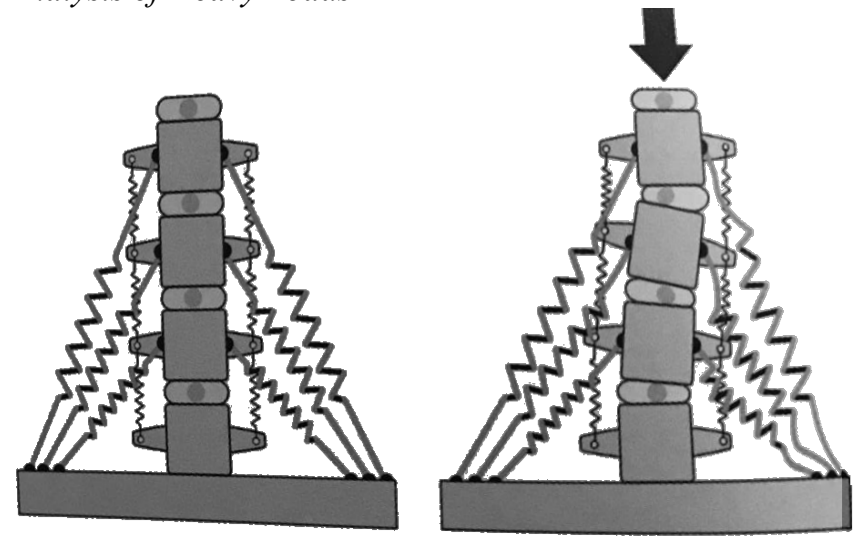

Fig. 3. How Back Strain occurs within a spring tensor model when large weights are carried incorrectly [13].

Research from the Wroclaw Medical University has identified a need to develop posture analysis systems for healthcare employees. The rise in back injuries with greater than $25 \mathrm{~kg}$ isare caused by-frem incorrect lifting practice. This leads to greater employee downtime and compensation related costs rising in many countries worldwide [14]. These human and financial costs are expected to increase as the population ages in many societies, placing greater pressure on healthcare workers to serve the community in time to come. The only true way to solve this emerging problem is through effective training protocols to enforce positive lifting practice in medical centers, regional clinics and metropolitan hospitals.

Thus as patient care work tasks rise in time as the median age of many countries increase, the design of an effective healthcare worker tool needs to be deployed offsite where AI analysis cannot always be performed on the premises, leading to a need for IoT driven solutions coupled with biomimetic middleware to manage motion tracking solutions effectively and seamlessly. Training resources must be managed in such a way to ensure the productivity of healthcare employees is consistently maintained to a positive standard.

The IoT biomimetic middleware platform must seek to summarize and encapsulate the possible human motion postures involved in the carrying process, mainly the lifting of patients and other heavy objects. The MLaaS/AIaaS backend needs to encapsulate a comprehensive databank that captures the historical key data points where injury risk is greatest in medical facilities. Key data points to be captured by the IoT motion capture framework include when [15]:

- $\quad$ The staff worker initially needs to prepare bending towards the patient;

- The staff worker adjusts their gait to obtain a better grip on the patient; and

- The load bearing stage as the staff worker lifts the patient, the act of transference, and

- Finally, the placement the patient onto another source as a wheelchair, stretcher or another bed.

The risks of Repetitive Strain Injury (RSI) rise dramatically as healthcare workers adopt poor or improper lifting practice, such that long term training and monitoring is necessary to ensure lifting techniques are evaluated at regular intervals - in order to test the candidate health workers' capabilities and technique from a progressive, longitudinal standpoint [13-14].

The IoT-driven platform should also encompass end-user consoles to allow healthcare workers to interact with the training platform, not only to communicate to remote diagnostics at the main training center, but also provide quantitative feedback as the lifting diagnosis takes place. This is important, as the wider adoption of the training platform can only take place when the healthcare worker physically views the result on their own viewing device, such as a connected smartphone or tablet, in active consultation and dialog with physiotherapy professionals.

Additionally $_{2}$ to the candidate healthcare worker quantitative analysis processes within the IOT-driven platform, the assessment needs to consider qualitative feedback as well. Hence, user interactivity design experts must be consulted to eventually design the end system to provide beneficial interaction with the candidate worker and physiotherapist as a means of constructive feedback and positive reinforcement outcomes.

The tool should not be seen as a punitive exercise on behalf of the healthcare institution, and thus ensuring that the continual liaison with main stakeholders is accomplished from a positive perspective. Any negative perception will defeat the 
purpose of the platform, so it is necessary to reinforce the core use case desires of healthy lifting practice, and achieve the main aim of healthcare worker morale enhancement and beneficial productivity engagement.

2) Coupling IoT-driven Biomimetics with Human Activity Recognition for Healthcare Scenarios

The main IoT backend concerns to consider is the comprehensive design and analysis of recognition algorithms tailed for human gait recognition. The classification of data sequences into movements that are well defined are essential, as well as the use of neural networks to offer state-of-the-art results on any challenging or complex gait recognition tasks that may take place within the AIaaS/MLaaS computational system layer.

Histogram of Gradients (HoG) approach is most commonly employed in such analysis concerns. The unique aspect of incorporating gait analysis for lifting contexts within an IoT biomimetic middleware design, would be to separate on-field with off-site data processing concerns to improve platform mobility, especially when deploying the system in remote medical clinics where available Internet access is limited or unstable.

The preparation and loading of gait recognition data, classified by time series, is then undertaken to allow the visualization and exploration of such data to the end user in liaison with the consultant physiotherapist. Therefore, the problem can be framed within an easy-to-use interface to allow the user to evaluate the results, and provide a contextual reference for the use case of best practices for manual patient lifting.

In addition, the concern of determining the kinematic activity of the healthcare worker, when human subjects interact within a specific space. Further investigation of the use of neural network or support vector machine approaches is of interest when the human subject performs a specific maneuver and needs to adjust their positioning for a given context.

\section{CONCLUSION AND FURTHER WORK}

The research workss seeks to analyze and demonstrate the practical benefits of employing a biomimetic middleware approach to IoT infrastructures, along with the relationship between the on-site and off-site machine learning concerns, The quality of the data processing concerns when it comes to IoT with motion capture needs to evaluate both quantitative user concerns, along with qualitative needs that the end user will also encounter in such an environment.

The needs of the end user is quality attribute that the design of the IoT biomimetic middleware must accommodate, in order to deal with various user concerns. By understanding the domain context, the design of the IoT middleware needs to compose of both managing the technical concerns of integrating heterogeneous IoT devices, while also being accommodating to the end user needs of usability and reliability. This will ensure the wider adoption of the middleware for a variety of IoT driven contexts and system frameworks.

Biomimetic principles in the design of the IoT middleware architecture must provide the essential framework of linking various components within a user-centric context, while also accommodating to future technological advances that play an important role in system design - this is examined in depth by the case study examined in this research paper with the rise in ubiquitous motion capture hardware.

The research case study seeks to highlight the need for a common biomimetic middleware framework for IoT mainline principles, and the main design factors that drive IoT into the future. The information flow from the architectural design, through to biomimetic design, shows that IoT plays a critical role as next generation mobile networks reach the mainstream economic sectors.

Hence, the understanding of suitable approaches, particularly learning-based ones like genetic algorithms and neural networks, along with the training data fed into these algorithms, is are-having real-world impacts into the operational capabilities of modern multimodal concerns. Also, of significant importance are, as well as the future trends influencing biomimetic design for performance optimization interests within the IoT connected domain.

\section{REFERENCES}

[1] Bantz D. F. et al, (2003) Autonomic personal computing, Autonomic Computing, Vol. 42, Number 1.

[2] Britton C., Bye P. (2004) "IT Architectures and Middleware: Strategies for Building Large, Integrated Systems", Addison Wesley Professional.

[3] Chaczko, Z.; Chiu, C.; Aslanzadeh, S.; Dune, T. (2011), Software Infrastructure for Wireless Sensor and Actuator Networks, Proceedings of 21 st International Conference on Systems Engineering, ICSEng2011, Las Vegas, pp.474-479.

[4] Chaczko, Z.C. \& Aslanzadeh, S. (2011), 'C2EN: Anisotropic Model of Cloud Computing', International Conference on Systems Engineering, Proceedings of 21 st Intl Conference on Systems Engineering, ICSEng 2011, Las Vegas, USA, pp.467-473.

[5] Chaczko, Z., (2009) "Towards Epistemic Autonomy in Adaptive Biomimetic Middleware for Cooperative Sensornets". PhD thesis, University of Technology, Sydney, Australia, July 2009.

[6] Clements, Paul; Felix Bachmann; Len Bass; David Garlan; James Ivers; Reed Little; Paulo Merson; Robert Nord; Judith Stafford (2010). Documenting Software Architectures: Views and Beyond, Second Edition. Boston: Addison-Wesley. ISBN 978-0-321-55268-6

[7] Bass, Len; Paul Clements; Rick Kazman (2012). Software Architecture in Practice, Third Edition. Boston: Addison-Wesley. ISBN 978-0-32181573-6

[8] M. Godinez, E. Hechler, K. Koenig, S. Lockwood, M. Oberhofer, M. Schroeck, The Art of Enterprise Information Architecture: A SystemsBased Approach for Unlocking Business Insight, IBM Press, 2010

[9] Krakowiak, S. Middleware Architecture with Patterns and Frameworks, 2009

[10] Steve McConnell, After The Goldrush - Creating a True Profession of Software Engineering, Microsoft Press, 1999

[11] Pressman et al, Software Engineering A Practitioner's Approach, Fifth Edition, McGraw Hill

[12] SEI, SE-CMM, The Systems Engineering Competency Maturity Model Architecture Papers, Carnegie Mellon University

[13] Carneiro, P., Braga, A.C. \& Barroso, M. (2017), Work-related musculoskeletal disorders in home care nurses: Study of main risk factors, International Journal of Industrial Ergonomics, 61: 22-28, https://doi.org/10.1016/j.ergon.2017.05.002

[14] European Council Directive 90/269/EEC, European Union Health and Safety Requirements for Manual Load Handling. https:/eurlex.europa.eu/legal-content/EN/TXT/?uri=CELEX\%3A31990L0269

[15] Health and Safety Executive, UK Government, Moving and Handling Case Studies in Health and Social Care http://www.hse.gov.uk/healthservices/moving-handling-case-studiesresearch.htm 